Eos, Transactions, American Geophysical Union, Vol. 74, No. 43, October 26, 1993, Page 493.

\section{Using Thermal Compression Waves to Assess Latent Heating from Clouds}

Meteorologists have extensively explored the dynamics of various inertial and gravity wave motions in the atmosphere [Holton, 1992; Haltiner and Williams, 1980]. These motions are assumed to be essentially incompressible, with the influence of sound waves being unimportant. Except for thunder and other propagation of audible sound waves, compression effects in the atmosphere have been generally ignored. Recently, a new theoretical framework has been developed which shows that thermally produced compression waves are critical to our understanding of atmospheric adjustment processes and the total energy budget. The thermally induced waves may provide valuable indicators of convective processes. Referred to as thermal compression/expansion waves, this wave energy occurs whenever diabatic heat sources or sinks occur as discussed by Nicholls and Pielke [1993a, 1993b].

The generation of a thermal compression wave can be described most simply for an idealized, horizontally homogeneous layer of the atmosphere whose bottom boundary is the ground. If it is assumed that the pressure is constant on this surface and heat is added either through the surface or by other diabatic effects such as latent heating or radiative flux convergence, the atmospheric layer must expand. This expansion is the thermal compression/expansion wave and occurs at the speed of sound. The amount of expansion is directly related to the magnitude of the diabatic heat input. However, the pressure at the surface cannot fall for this idealized example, because no mass has been removed from the horizontally homogeneous layer.

Until recently, it seemed that there was no practical way to measure this acoustic wave. The acoustic sampling network developed by Einaudi et al. [1989], however, provides a unique opportunity to sample this type of atmospheric disturbance.

Using arrays of low-frequency, infrasonic microphones or sensitive microbarographs, it is possible to determine the direction, phase speed, and spectral characteristics of atmospheric acoustic waves with frequencies in the range of $20 \mathrm{~Hz}$ to less than $.01 \mathrm{~Hz}$. Although pressure amplitudes of measured acoustic waves are quite small (of the order of $10^{-6}$ of an atmosphere), digital real-time processing techniques as well as spatial noise reduction filters [e.g., Bedard, 1977, 1988] permit essentially continuous detection at distances from tens to thousands of kilometers from the source, except possibly during strong local wind conditions. The measured powers from infrasonic waves from severe weather are in the range of $10^{7}$ $w$. The development of the theoretical framework reported in Nicholls and Pielke [1993a, 1993b] permits interpretation of the low-frequency acoustic measurements in terms of the convective processes that caused them. On the one hand, it will be valuable to design an experiment to detect the long period of thermal waves. In addition, it will be valuable to extend the numerical simulations to include shorter-period acoustic waves, which may also provide an important index of storm severity.

Since the intensity of the thermal compression waves is directly related to the amount of latent heat release, it is feasible to establish two separate in-space sampling networks in order to triangulate on a developing cumulus cloud. The direction from which the compression wave is propagating will be different at the two sampling sites, so that the geographic location of the heat released by the cumulus cloud can be determined. By assessing the thermal compressible energy that is generated by this cloud, it should be possible to diagnose the time evolution of the latent heating from this atmospheric system. This is an entirely new approach to assess the intensity of developing cumulus cloud features and may provide us with a technique to independently evaluate the severity of thunderstorms.

Acknowledgments: This work was supported under NSF Cooperative Agreement \#BCS-8821542 with Texas Tech University and NSF Grant \#ATM-8915265. Dallas McDonald and Bryan Critchfield ably edited and prepared the paper.- $R$. A. Pielke and M. E. Nicholls, Colorado State University, Fort Collins; and A. J. Bedard, NOAA, Boulder, Colo.

\section{References}

Bedard, A. J., Jr., Infrasound from natural resources, Inter-Noise 88, Avingnon, France, 30 August, 1988, 927-930.

Bedard, A. J., Jr., The D-C pressure summator: Theoretical operation, experimental tests, and possible practical uses, Fluidics Quart., 1, 26, 1977.

Einaudi, F. A. J. Bedard, Jr., and J. J. Finnigan, A climatology of gravity waves and other coherent disturbances at the Boulder atmospheric observatory during March-April 1984, J. Atmos. Sci., 46, 303, 1989.

Holton, J. R., An Introduction To Dynamic Meteorology, Academic Press, Orlando, Fla., 497 pp., 1992.

Haltiner, G. J., and R. T. Williams, Numerical Prediction and Dynamic Meteorology, Wiley and Sons, Inc., New York, 477 pp., 1980.

Nicholls, M. E., and R. A. Pielke, Thermal compression waves. Part I: Total energy transfer, $Q$. J. R. Meteorol. SoC., in press, 1993a.

Nicholls, M. E., and R. A. Pielke, Thermal compression waves. Part II: Mass adjustment and vertical transfer of total energy, Q.J. R. Meteorol. Soc. in press, 1993b. 